\title{
EXTENSIONS OF A RING BY A RING WITH A BIMODULE STRUCTURE
}

\begin{abstract}
CARL W. KOHLS
ABSTRACT. A type of ring extension is considered that was introduced by J. Szendrei and generalizes many familiar examples, including the complex extension of the real field. We give a method for constructing a large class of examples of this type of extension, and show that for some rings all possible examples are obtained by this method. An abstract characterization of the extension is also given, among rings defined on the set product of two given rings.
\end{abstract}

This paper is a sequel to [2], in which a class of examples was given of a type of ring extension defined in terms of two functions. Here we exhibit some other functions that may be used to construct such extensions, and show that in certain cases (in particular, when the first ring is an integral domain and the second is a commutative ring with identity), the functions must have a prescribed form. We also characterize this type of ring extension, which Szendrei defined directly by the ring operations, in terms of the manner in which the two given rings are embedded in the extension.

The reader is referred to [2] for background. Only the basic definition is repeated here.

Let $A$ and $B$ be rings. We define the ring $A * B$ to be the direct sum of $A$ and $B$ as additive groups, with multiplication given by

$$
(a, b)(c, d)=\left(a c+\{b, d\}, \sigma_{a} d+b \sigma_{c}+b d\right),
$$

where $\sigma$ is a homomorphism from $A$ onto a ring of permutable bimultiplications of $B$, and $\{\cdot, \cdot\}$ is a biadditive function from $B \times B$ into $A$ satisfying the equations

$$
\begin{gathered}
b \sigma_{\{c, d\}}=\sigma_{\{b, c\}} d, \\
\{b, c d\}=\{b c, d\}, \\
\left\{b, \sigma_{a} c\right\}=\left\{b \sigma_{a}, c\right\}, \\
\left\{\sigma_{a} b, c\right\}=a\{b, c\}, \text { and } \quad\left\{b, c \sigma_{a}\right\}=\{b, c\} a,
\end{gathered}
$$

for all $a \in A$ and all $b, c, d \in B$. As noted in [2], when $B$ has an identity, (4) is redundant. The inverse of a homomorphism $\phi$ will be denoted by $\phi^{\prime}$, even when $\phi$ is not a monomorphism.

Received by the editors September 2, 1969.

AMS Subject Classifications. Primary 1680, 1380; Secondary 1315.

Key Words and Phrases. Ring extension, bimultiplication ring, biadditive function, bimodule, commutative ring with identity, integral domain. 
We first characterize those rings on the set product $A \times B$ that have the form $A * B$, making only one natural assumption about the addition operation. The following concept will be required.

Definition 1. Let $A, B$, and $E$ be rings and let $i: A \rightarrow E, j: B \rightarrow E$ be injections. If $j(b+d)=j(b)+j(d)$ and $j(b) j(d)-j(b d) \in i(A)$ for all $b, d \in B$, we say that $B$ is embedded in $E$ as a subring modulo $A$.

Proposition 1. Let $A$ and $B$ be rings, and assume that $A \times B$ has been made into a ring $E$ such that $(a, b)=(a, 0)+(0, b)$ for all $(a, b)$ $\in A \times B$. Then $E$ has the form $A * B$ if and only if $A$ is embedded as a subring and $B$ is embedded as an $A$-bimodule and as a subring modulo $A$.

Proof. It is evident that if $E$ has the form $A * B$, then $A$ is embedded as a subring and $B$ as an abelian group. Since $(a, 0)(0, b)$ $=\left(0, \sigma_{a} b\right)$ and $(0, b)(a, 0)=\left(0, b \sigma_{a}\right)$ for all $a \in A$ and $b \in B$, it follows from the associative and distributive laws in $A * B$ that $B$ is embedded as an $A$-bimodule; and since $(0, b)(0, d)=(\{b, d\}, 0)+(0, b d)$ for all $b, d \in B$, it is embedded as a subring modulo $A$.

For the converse, first note that for all $a, c \in A$ and $b, d \in B$, $(a, b)+(c, d)=(a, 0)+(0, b)+(c, 0)+(0, d)=(a+c, 0)+(0, b+d)$ $=(a+c, b+d)$, and $(a, 0)(c, 0)=(a c, 0),(a, 0)(0, d)=(0, f(a, d))$, $(0, b)(c, 0)=(0, g(b, c)),(0, b)(0, d)=(h(b, d), 0)+(0, b d)=(h(b, d), b d)$ for some functions $f, g, h$, so that $(a, b)(c, d)=(a, 0)(c, 0)+(a, 0)(0, d)$ $+(0, b)(c, 0)+(0, b)(0, d)=(a c+h(b, d), f(a, d)+g(b, c)+b d)$. The associative and distributive laws in $E$ imply that the functions $f$ and $g$ yield a homomorphism $\sigma$ from $A$ onto a ring of permutable bimultiplications of $B$, defined by $\sigma_{a} d=f(a, d), b \sigma_{c}=g(b, c)$. These laws also yield the facts that $h$ is a biadditive function and that (2)-(5) are satisfied. Hence we may write $h(b, d)=\{b, d\}$, and $(a, b)(c, d)$ $=\left(a c+\{b, d\}, \sigma_{a} d+b \sigma_{c}+b d\right)$. Thus, $E$ has the form $A * B$.

Remarks. 1 . Of course, the $A$-bimodule and ring multiplications on $B$ are related by the associative law in $E$, as seen in the proof.

2. If it is not assumed that $B$ is embedded as a subring modulo $A$, one has $(0, b)(0, d)=(h(b, d), k(b, d))$ for some functions $h, k$. It follows that $k$ is biadditive; but it is not possible to show that $k$ is associative unless (2) is assumed, since both identities can be deduced only from a single equation implied by the associativity of the product $(0, b)(0, c)(0, d)$.

3. If $B$ is actually embedded as a subring, then it is embedded as an ideal. For in this case $\{\cdot, \cdot\} \equiv 0$, so we get a splitting Everett extension.

Some generality is gained in our main result by use of the following concept. 
Definition 2. We say that two rings $B$ and $(B, \cdot)$ with the same additive group and multiplications $(b, c) \mapsto b c,(b, c) \mapsto b \cdot c$ are compatible if $(b \cdot c) d=b \cdot(c d)=(b c) \cdot d=b(c \cdot d)$ for all $b, c, d \in B$.

EXAMPLE 1. Let $p$ be a fixed element in the center of a given ring $B$, and let $B_{p}$ denote, as in [2], the ring with the same additive group as $B$ and with multiplication defined by the mapping $(b, c) \mapsto p b c$. Then $B$ and $B_{p}$ are compatible. In particular, $B$ and the zero-ring $B_{0}$ are compatible.

TheOREM. Let $A$ and $B$ be rings, $(B, \cdot)$ a ring compatible with $B$, and $\phi: A \rightarrow B$ a homomorphism. Let $s$ be an element in the center of $B$ such that $(s) \subseteq \operatorname{Im} \phi$, and $q$ an element in the center of $\phi^{\prime}((s))$ and in the annihilator of $\operatorname{Ker} \phi$. Define $\sigma$ by

$$
\sigma_{a} b=\phi(a) b, \quad b \sigma_{a}=b \phi(a), \text { for all } a \in A, \quad b \in B,
$$

and $\{\cdot, \cdot\}$ by

$$
\{b, c\}=q \phi^{\prime}(s b c), \text { for all } b, c \in B .
$$

Then with multiplication defined by (1), one obtains a ring extension $A *(B, \cdot)$.

Conversely, if $B$ is a ring with identity, and a ring extension $A * B$ is given, then $\sigma$ is defined by (6) for some homomorphism $\phi: A \rightarrow B$. Furthermore, if (I) $\phi$ is an epimorphism, then $\{\cdot, \cdot\}$ is defined by (7), with $q=\{1,1\}$ and $s=1$; or if (II) $B$ is commutative and there exist $q, r \in A$ such that $r q=\{1,1\},(\phi(r)) \subseteq \operatorname{Im} \phi$, and $r$ is not a zero-divisor, then $\{\cdot$,$\} is defined by (7), with s=\phi(r)$. In either case, $q$ is in the center of $\phi^{\prime}((s))$ and in the annihilator of $\operatorname{Ker} \phi$.

Proof. Clearly, $\sigma$ as defined in (6) is a homomorphism of $A$ onto a ring of permutable bimultiplications of $(B, \cdot)$, and $\{\cdot, \cdot\}$ as defined in (7) is well-defined and biadditive. Noting that $\phi(q)$ is in the center of $(s)$, one checks easily that (2)-(5) are satisfied, so one obtains a ring extension $A *(B, \cdot)$ by using these functions in (1).

Now if $B$ is a ring with identity it follows from $[1$, Proposition 4$]$ that every bimultiplication of $B$ is inner; and if a ring extension $A * B$ is given, then $1\left(\sigma_{a} 1\right)=\left(1 \sigma_{a}\right) 1$ for each $a \in A$, so $\sigma_{a}$ is multiplication on the left and right by the same element of $B$. Hence $\sigma$ is defined by (6) for some homomorphism $\phi: A \rightarrow B$.

Assume that $\phi$ is an epimorphism, and for $b, c \in B$ choose $b^{\prime} \in \phi^{\prime}(b)$ and $c^{\prime} \in \phi^{\prime}(c)$. By (5), $b^{\prime}\{1,1\} c^{\prime}=\left\{\sigma_{b^{\prime}} 1,1 \sigma_{c^{\prime}}\right\}=\{b 1,1 c\}=\{b, c\}$, whence $\{b, c\}=b^{\prime} q c^{\prime}$ with $q=\{1,1\}$. A similar calculation gives $\{1, b\}=q b^{\prime},\{b, 1\}=b^{\prime} q$; but by $(3),\{1, b\}=\{b, 1\}$, so $q b^{\prime}=b^{\prime} q$, and $q$ is in the center of $A=\phi^{\prime}((1))$. Since $\{1, b\}=q b^{\prime}=b^{\prime} q$ for any choice 
of $b^{\prime}$, and $\{1, b\}$ is well-defined, $q$ is in the annihilator of $\operatorname{Ker} \phi$, and we may write $\{b, c\}=q \phi^{\prime}(b c)$.

Now assume that $B$ is commutative and there exist $q, r \in A$ as in (II). For $b, c \in B$, choose $d^{\prime} \in \phi^{\prime}(s b c)$. Then $r\{b, c\}=\{\phi(r) b, c\}$ $=\{1, s b c\}=\{1,1\} d^{\prime}=r q d^{\prime}$, whence $\{b, c\}=q d^{\prime}$. Thus $q$ is in the left annihilator of $\operatorname{Ker} \phi$, and $\{b, c\}=q \phi^{\prime}(s b c)$, that is, (7) holds. Since (7) yields $\{1,1\}=q \phi^{\prime}(s)=q r$, we also have $\{b, c\} r=\{b, c \phi(r)\}$ $=\{s b c, 1\}=d^{\prime}\{1,1\}=d^{\prime} q r$. Hence $\{b, c\}=d^{\prime} q$, and so $d^{\prime} q=q d^{\prime}$. Therefore $q$ is in the right annihilator of $\operatorname{Ker} \phi$ and in the center of $\phi^{\prime}((s))$.

Corollary. Let $A$ be an integral domain, and $B$ a commutative ring with identity. In any ring extension $A * B, \sigma$ is defined by (6) for some homomorphism $\phi: A \rightarrow B$. If $\{\cdot, \cdot\} \equiv 0$, then $\{\cdot, \cdot\}$ is defined by (7), with $q, s=0$; if $\{\cdot, \cdot\} \not \equiv 0$, then $\phi$ is a monomorphism and $\{\cdot, \cdot\}$ is defined by (7), with $q=1$ and $s=\phi(\{1,1\})$.

Proof. The assertion about $\sigma$ is contained in the theorem, and the first statement about $\{\cdot, \cdot\}$ is obvious. If $\phi$ is not a monomorphism, choose any nonzero $a \in \operatorname{Ker} \phi$. Then for all $b, c \in B$ we have $a\{b, c\}$ $=\{\phi(a) b, c\}=0$, whence $\{b, c\}=0$. Now assume that $\{\cdot, \cdot\} \not \equiv 0$. Since $\{b, c\}=\{1, b c\}$, there is some $d \in B$ for which $\{1, d\} \neq 0$. By (2), $\phi(\{1,1\}) d=\phi(\{1, d\}) \neq 0$, because $\phi$ is a monomorphism; hence $\{1,1\} \neq 0$. Set $r=\{1,1\}$ and $q=1$. Then $\phi(r) b=\phi(\{1,1\}) b=\phi(\{1, b\})$ for all $b \in B$, so $(\phi(r)) \subseteq \operatorname{Im} \phi$. The theorem now shows that $\{\cdot, \cdot\}$ is defined by (7), with $q=1$ and $s=\phi(\{1,1\})$.

Remarks. 4. A slight generalization of the last part of the proof of the corollary shows that if $\phi(r) \in \operatorname{Im}(\phi \mid \operatorname{Im}\{\cdot, \cdot\})$, then $(\phi(r)) \subseteq \operatorname{Im} \phi: \phi(\{b, c\}) d=\phi(\{1, b c\}) d=\phi(\{b c, d\})$ for all $b, c, d \in B$, so any $r \in A$ such that $\phi(r)=\phi(\{b, c\})$ satisfies $(\phi(r)) \subseteq \operatorname{Im} \phi$.

5. Obviously if $\operatorname{Im} \phi$ is itself an ideal, it is easier to find an $r$ satisfying hypothesis (II) of the theorem. In fact, if $A$ has an identity, we can pick $r=1$.

We now give several examples of ring extensions $A *(B, \cdot)$ by specifying the homomorphism $\phi$ and elements $q, s$ to be used in (6) and (7). Except in Example 6, $(B, \cdot)=B$.

ExAmples. 2. Let $A$ be the algebra over the real field $R$ generated by an identity element 1 and an element $a$ satisfying $a^{2}=0$, and let $B=R \oplus R$. Define $\phi$ by $\phi(t+u a)=(t, 0)$ for $t, u \in R$, and choose $q=a$, $s=(1,0)$. Note that $\left\{\left(t_{1}, u_{1}\right),\left(t_{2}, u_{2}\right)\right\}=t_{1} t_{2} a$ and $\operatorname{Im} \phi$ is an ideal. To verify that hypothesis (II) of the theorem holds, one could choose $r=1$. 
A similar example in which $\phi$ is an epimorphism is obtained by simply setting $B=R$.

3. Let $A$ be the ring of all continuous real-valued functions on $[-1,1]$ assuming an even integer value at 1 , and $B$ the ring of all continuous real-valued functions on $[0,1]$ assuming an integer value at 1 . Let $\phi$ be induced by restriction of functions to $[0,1], s$ be the constant function 2 on $[0,1]$, and $q$ be defined on $[-1,1]$ by $q(t)=0$ for $t \leqq 0$ and $q(t)=2 t$ for $t \geqq 0$. Note that $\{b, c\}$ satisfies $\{b, c\}(t)=0$ for $t \leqq 0$ and $\{b, c\}(t)=4 t b(t) c(t)$ for $t \geqq 0$. Again $\operatorname{Im} \phi$ is an ideal, and here one could choose $r$ to be the constant function 2 on $[-1,1]$.

4. Let $B$ be the ring of all polynomials in $x$ over the real field, $A$ the subring of polynomials with rational constant term, $\phi$ the natural injection, $q=1$ and $s=x$. Note that $\{b, c\}=x b c$ and $\operatorname{Im} \phi$ is not an ideal.

5. Let $A$ be the ring of integers and $B$ any field of characteristic not 2 . In any ring extension $A * B$, we must have $\{\cdot, \cdot\} \equiv 0$ : Otherwise, if $\{b, c\}$ were the least positive integer in $\operatorname{Im}\{\cdot, \cdot\}$, we should have $\{b / 2, c\}=(\{b, c\}) / 2$, a contradiction. Thus in any ring extension $A * B,\{\cdot, \cdot\}$ may be written as in (7), with $q, s=0$, even though hypotheses (I) and (II) of the theorem both fail to hold when the characteristic of $B$ is 0 . (Compare this with the corollary and its proof.)

6. Let $A$ be the ring of all real-valued functions on a topological space $X, 1+q$ the characteristic function of a nonempty subset $Z$ of $X, B$ the ring of all real-valued functions on $X-Z$, and $B_{0}$ the zeroring with the same additive group as $B$. Let $\phi$ be the homomorphism induced by restriction to $X-Z$, and choose $s$ to be the constant function 1 on $X-Z$. Each element of $A * B_{0}$ naturally determines a function on $X-Z$. Examining the addition and multiplication in the ring obtained from $A * B_{0}$ by evaluation of these functions at a point of $X-Z$, we see that the functions on $X-Z$ determined by the elements of $A * B_{0}$ are complex-valued. Let $C$ be the subring of $A$ of continuous functions on $X$, and $D$ the subring of $B_{0}$ of continuous functions that approach 0 on the boundary of $X-Z$ in $X$. Using the same ring operations on $C \times D$, one obtains a subring of $A * B_{0}$, and it may be viewed as the ring of continuous complex-valued functions on $X$ that are real-valued on $Z$.

The last example shows that functions defined by (6) and (7) do not yield directly a simple class of ring extensions, and suggests consideration of some slightly different functions.

Proposition 2. Let $B$ be an ideal in a ring $A, B_{p}$ any compatible ring as in Example 1, $\phi$ an automorphism of $A$, and $q$ an element of $A$ 
that commutes with each element of $\operatorname{Im}\left(\phi^{\prime} \mid B\right)$. Define $\sigma$ by

$$
\sigma_{a} b=\phi(a) b, \quad b \sigma_{a}=b \phi(a), \text { for all } a \in A, b \in B,
$$

and $\{\cdot, \cdot\}$ by

$$
\{b, c\}=q \phi^{\prime}(b c), \text { for all } b, c \in B .
$$

Then with multiplication defined by (1), one obtains ring extensions $A * B$ and $A * B_{p}$.

The proof is again a straightforward verification.

ExAmple 7. Let $A$ be the ring of all continuous real-valued functions on a topological space $X, B$ the ideal of all functions in $A$ that vanish on a fixed nonempty subset $Z$ of $X, \phi$ the identity automorphism on $A$, and $q$ the constant function -1 on $X$. Then with $\sigma$ and $\{\cdot, \cdot\}$ defined by (8) and (9), $A * B_{0}$ is isomorphic to the ring of continuous complex-valued functions on $X$ that are real-valued on $Z$.

\section{REFERENCES}

1. C. W. Kohls and L. J. Lardy, On extensions and bimultiplication algebras of algebras, Arch. Math. 20 (1969), 365-372.

2. - Some ring extensions with matrix representations, Pacific J. Math. 26 (1968), 341-348. MR 38 \#5853.

Syracuse University, Syracuse, New York 13210 